\title{
Dynamics of the entanglement between two oscillators in the same environment
}

\author{
Juan Pablo Paz ${ }^{1}$ and Augusto J. Roncaglia ${ }^{1}$ \\ ${ }^{1}$ Departamento de Física, FCEyN, UBA, Pabellón 1, \\ Ciudad Universitaria, 1428 Buenos Aires, Argentina
}

\begin{abstract}
We provide a complete characterization of the evolution of entanglement between two oscillators coupled to a common environment. For initial Gaussian states we identify three phases with different qualitative long time behavior: There is a phase where entanglement undergoes a sudden death (SD). Another phase (SDR) is characterized by an infinite sequence of events of sudden death and revival of entanglement. In the third phase (NSD) there is no sudden death of entanglement, which persist for long time. The phase diagram is described and analytic expressions for the boundary between phases are obtained. Numerical simulations show the accuracy of the analytic expressions. These results are applicable to a large variety of non-Markovian environments. The case of non-resonant oscillators is also numerically investigated.
\end{abstract}

Entanglement is responsible for the most counterintuitive aspects of quantum mechanics. In the early days of quantum physics, entanglement motivated many philosofical discussions but nowadays it is viewed merely as a source of surprises but, mainly, as a physical resource. In fact, entanglement is the key ingredient for quantum teleportation and is believed to be the origin of the power of quantum computers. Systems of qubits interacting with common or independent environment where extensively analyzed in the literature $[1,2,2,3,4]$. In such context, the existence of a peculiar property was noticed in [5]: Entanglement, as opposed to energy, can disappear from a system in a finite time (this phenomenon was denoted as "sudden death" SD of entanglement).

Here we study the evolution of the entanglement between two oscillators interacting with a common bosonic environment. Entanglement dynamics for such kind of systems was analyzed before and a variety of results is available in the literature. For example: It was shown that the environment may completely dissentangle initially entangled state (a two-mode squeezed state). Thus, in [6, 7, 8] the existence of sudden death was proved and analyzed for such case. The evolution of the same type of initially entangled state was analyzed when the two oscillators interact with a common bath under a simplifying assumption: the Markovian approximation 9, 10, 11]. In that case, a condition for the existence of an asymptotic entangled state was deduced [9]. If such condition is not satisfied (see below) sudden death takes place. Also, it was shown that the interaction with a common environment opens the door to the creation of an entangled state from an initial separable state [11]. Most of these works were done under some sort of Markovian approximation. More recently, the non-Markovian regime was analyzed [12, 13, 14]. Under special conditions a simple result emerged: The final state remains entangled but half of the initial entanglement is lost [13].

Our work provides a unified picture to understand the origin of the different qualitative behaviors ("phases") that characterize the evolution of entanglement for long times. We will fully characterize these phases of the evo- lution and obtain equations describing the boundary between them. For this purpose we will use a well known tool: the exact master equation for quantum Brownian motion [15]. We consider two harmonic oscillators $\left(x_{1}\right.$ and $x_{2}$ ) bilinearly coupled between them and with a collection of harmonic oscillators [15, 16, 17, 18]. The total Hamiltonian is $H=H_{S}+H_{\text {int }}+H_{\text {env }}$ where

$$
\begin{aligned}
H_{S} & =\frac{p_{1}^{2}+p_{2}^{2}}{2 m}+\frac{1}{2} m\left(\omega_{1}^{2} x_{1}^{2}+\omega_{2}^{2} x_{2}^{2}\right)+m c_{12} x_{1} x_{2} \\
H_{\text {env }} & =\sum_{n=1}^{N}\left(\frac{\pi_{n}^{2}}{2 m_{n}}+\frac{m_{n}}{2} w_{n}^{2} q_{n}^{2}\right), \\
H_{\text {int }} & =\left(x_{1}+x_{2}\right) \sum_{i=1}^{N} c_{n} q_{n} .
\end{aligned}
$$

Using coordinates $x_{ \pm}=\left(x_{1} \pm x_{2}\right) / \sqrt{2}$, the Hamiltonian transforms into $H_{S}=\left(p_{+}^{2}+p_{-}^{2}\right) / 2 m+m\left(\omega_{-}^{2} x_{-}^{2}+\right.$ $\left.\omega_{+}^{2} x_{+}^{2}\right) / 2+m c_{+-} x_{+} x_{-}$, where $\omega_{ \pm}^{2}=\left(\omega_{1}^{2}+\omega_{2}^{2}\right) / 2 \pm c_{12}$ and $c_{+-}=\left(\omega_{1}^{2}-\omega_{2}^{2}\right) / 2$. This model can be exactly solved assuming that the initial state of the environment is thermal with initial temperature $T$ [15], and entanglement properties between the system and the environment were studied in [19]. The resonant case $\left(\omega_{1}=\omega_{2}\right)$, where the $x_{ \pm}$oscillators are decoupled, is the simplest: The exact master equation for the reduced density matrix of the two oscillators $\rho$ is $(\hbar=1)[15,18]$ :

$$
\begin{aligned}
\dot{\rho} & =\frac{1}{i}\left[H_{R}, \rho\right]-i \gamma(t)\left[x_{+},\left\{p_{+}, \rho\right\}\right]- \\
& -D(t)\left[x_{+},\left[x_{+}, \rho\right]\right]-f(t)\left[x_{+},\left[p_{+}, \rho\right]\right] .
\end{aligned}
$$

Here, the renormalized Hamiltonian is $H_{R}=H_{S}+$ $m \delta \omega^{2}(t) x_{+}^{2} / 2$. The coefficients $\delta \omega^{2}(t), \gamma(t), D(t)$ and $f(t)$ depend on the spectral density of the environment, defined as $J(\omega)=\sum_{n} c_{n}^{2} \delta\left(\omega-w_{n}\right) / 2 m_{n} w_{n}$, and also on its initial temperature. The explicit form of these coefficients is rather cumbersome and was studied in detail elsewhere [15, 20]. We will focus on the ohmic environment with a spectral density $J(\omega)=2 m \gamma_{0} \omega \theta(\omega-\Lambda) / \pi$. The high frequency cutoff $\Lambda$ defines a characteristic 
timescale $\Lambda^{-1}$ over which the coefficients $\gamma(t)$ and $\delta \omega^{2}(t)$ vary. For times $t \gg \Lambda^{-1}$ these two temperature independent coefficients settle into asymptotic values: $\gamma(t) \rightarrow$ $\gamma=2 \gamma_{0}$ and $\delta \omega^{2}(t) \rightarrow-4 m \Lambda \gamma / \pi$. The time dependent frequencies $\Omega_{1,2}^{2}(t)=\omega_{1,2}^{2}+\delta \omega^{2}(t) / 2$ approach cutoff independent values only if the bare frequencies $\omega_{1,2}$ have an appropriate dependence on the cutoff. The coupling constant $c_{1,2}$ must also be renormalized in the same way so that the time dependent coupling $C_{12}(t)=c_{12}+\delta \omega^{2}(t) / 2$ approaches a finite cutoff independent value. The behavior of the diffusion coefficients $D(t)$ and $f(t)$ is more complicated and depend on the initial temperature. For the moment we just need to mention here that for realistic environments these coefficients approach asymptotic values after a temperature-dependent time (which equals $\Lambda^{-1}$ only in the high temperature regime). A word on notation: upper case letters will be used to denote renormalized quantities. The time label will be omitted when referring to the asymptotic value of the corresponding function (i.e., $\Omega_{1,2}$ denotes the asymptotic value of the renormalized frequency of the oscillators, etc).

From the master equation (2) we can derive simple evolution equations for the covariance matrix $V_{i j}(t)=$ $\operatorname{Tr}\left(\rho(t)\left\{r_{i}, r_{j}\right\}\right) / 2-\operatorname{Tr}\left(\rho(t) r_{i}\right) \operatorname{Tr}\left(\rho(t) r_{j}\right)$ where $i, j=$ $1, \ldots, 4$ and $\vec{r}=\left(x_{-}, p_{-}, x_{+}, p_{+}\right)$. Some of these equations are particularly illuminating: In fact, equations for the covariances split into two blocks of $2 \times 2$. The evolution of the first block formed with the second moments of $x_{-}$and $p_{-}$corresponds to a free oscillator with frequency $\Omega_{-}^{2}(t)$. The evolution of the second block, formed with the second moments of $x_{+}$and $p_{+}$, satisfys:

$$
\begin{aligned}
& \frac{d}{d t}\left(\frac{\left\langle p_{+}^{2}\right\rangle}{2 m}+\frac{m}{2} \Omega^{2}(t)\left\langle x_{+}^{2}\right\rangle\right)=-\frac{2 \gamma(t)}{m}\left\langle p_{+}^{2}\right\rangle+\frac{D(t)}{m}, \\
& \frac{1}{2} \frac{d^{2}\left\langle x_{+}^{2}\right\rangle}{d t^{2}}+\gamma(t) \frac{d\left\langle x_{+}^{2}\right\rangle}{d t}+\Omega^{2}(t)\left\langle x_{+}^{2}\right\rangle=\frac{\left\langle p_{+}^{2}\right\rangle}{m^{2}}-\frac{f(t)}{m} .
\end{aligned}
$$

These equations contain most of the necessary information to fully analyze the evolution of the entanglement between initial Gaussian states. To solve them exactly we need to know the time dependent coefficients that appear in the master equation. But, remarkably enough, we can use the above equations to understand the qualitative behavior of entanglement. For this, we only need to assume that the time dependent coefficients in (2) approach asymptotic values. In such case, there is a stable stationary solution where the dispersions $\Delta^{2} x_{+}=\left\langle x_{+}^{2}\right\rangle$ and $\Delta^{2} p_{+}=\left\langle p_{+}^{2}\right\rangle$ are

$$
\Delta p_{+}=\sqrt{\frac{D}{2 \gamma}} ; \quad \Omega \Delta x_{+}=\sqrt{\frac{D}{2 m^{2} \gamma}-\frac{f}{m}} .
$$

Entanglement for Gaussian states is entirely determined by the properties of the covariance matrix $V_{i j}$. In fact, a good measure of entanglement for such states is the logarithmic negativity $E_{\mathcal{N}}[21,22]$, computable as [21, 22, 23]:

$$
E_{\mathcal{N}}=\max \left\{0,-\ln \left(2 \nu_{\min }\right)\right\},
$$

where $\nu_{\min }$ is the smallest symplectic eigenvalue of the partially transposed covariance matrix. There are known expressions for $E_{\mathcal{N}}$ for particularly relevant Gaussian states which will be used as initial conditions in our study. For this reason it is useful to mention them here: For the two-mode squeezed state, obtained from the vacuum by acting with the operator $\exp \left(-r\left(a_{1}^{\dagger} a_{2}^{\dagger}-a_{1} a_{2}\right)\right)$, we have $E_{\mathcal{N}}=2|r|$. For this state the dispersions satisfy the minimum uncertainty condition $\delta x_{+} \delta p_{+}=\delta x_{-} \delta p_{-}=1 / 2$. The squeezing factor determines the ratio between variances since $m \Omega \delta x_{+} / \delta p_{+}=\delta p_{-} /\left(m \Omega \delta x_{-}\right)=\exp (2 r)$. As $r \rightarrow \infty$ the state becomes localized in the $p_{+}$and $x_{-}$ variables approaching an ideal EPR state 24]. Another initial state we will consider is a separable squeezed state for which $m \Omega \delta x_{1,2} / \delta p_{1,2}=\exp (2 r)$.

The evolution of entanglement for initial Gaussian states can then be simply analyzed by using the previous results. Let us consider a general initial state. For the resonant case, the $x_{-}$virtual oscillator decouples exactly. Using the equations (4) we see that $\Delta x_{+}$and $\Delta p_{+}$ approach asymptotic values after a timescale $1 / \gamma$, fixed by the dissipation rate. After that time, the covariance matrix in the $\left(x_{+}, x_{-}\right)$bases has a $2 \times 2$ block with oscillatory functions (corresponding to the $x_{-}$oscillator) and another $2 \times 2$ block, corresponding to the $x_{+}$virtual oscillator, which is diagonal. Using this form for the covariance matrix (and changing basis to obtain covariances of the original $x_{1,2}$ oscillators) it is simple to find its smallest symplectic eigenvalue and compute the following logarithmic negativity 25]

$$
E_{\mathcal{N}}(t) \rightarrow \max \left\{0, \tilde{E}_{\mathcal{N}}+\Delta E_{\mathcal{N}} G(t)\right\} .
$$

Where $G(t)$ is a function with period $\pi / \Omega_{-}$in $\{-1,1\}$. The mean value $\tilde{E}_{\mathcal{N}}$ about which $E_{\mathcal{N}}$ oscillates is

$$
\tilde{E}_{\mathcal{N}}=\max \left\{r, r_{\text {crit }}\right\}-\frac{1}{2} \ln \left(2 \Delta x_{+} \Delta p_{+}\right),
$$

and the amplitude of the oscillation is:

$$
\Delta E_{\mathcal{N}}=\min \left\{r, r_{\text {crit }}\right\} .
$$

Here $r$ is the initial squeezing factor defined as $r=\left|\frac{1}{2} \ln \left(m \Omega_{-} \frac{\delta x_{-}}{\delta p_{-}}\right)\right|, r_{c r i t}$ is related to the squeezing factor of the equilibrium state for the $x_{+}$-oscillator defined as $r_{c r i t}=\left|\frac{1}{2} \ln \left(m \Omega_{-} \frac{\Delta x_{+}}{\Delta p_{+}}\right)\right|$. The dispersions $\Delta x_{+}$and $\Delta p_{+}$are the asymptotic values (5).

These simple results will enable us to draw general conclusions about the dynamics of entanglement for long times. Three qualitatively different scenarios emerge: First, entanglement may persist for arbitrary long times. This phase, which we call "NSD" 

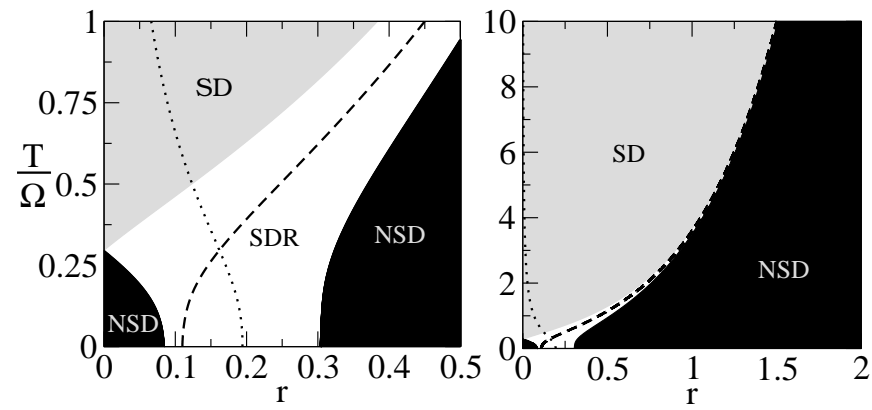

FIG. 1: Phase diagram for ohmic environment $(\Omega=1$, $\left.\gamma_{0}=0.15, \Lambda=20, m=1, C_{12}=0\right)$. The sudden death (SD), no-sudden death (NSD) and sudden death and revival (SDR) phases describe the three different qualitative long time behaviors for the entanglement between two oscillators interacting with the same environment. The SDR phase is centered about the dashed line $S_{r}$ and has a width given by the dotted line $r_{c r i t}$. This is the case for temperatures above the one for which $S_{r}=r_{\text {crit }}$. Below this temperature the role of $S_{r}$ and $r_{c r i t}$ are interchanged. SDR separates the SD and NSD phases. The low temperature NSD island is due to non-Markovian and non-perturbative effects. $\tilde{E}_{\mathcal{N}}$ in the NSD phase is the distance to the dashed line for $r>r_{c r i t}$, and the distance between the dashed and dotted lines for $r \leq r_{\text {crit }}$.

(for no-sudden death), is realized when the initial state is such that $\tilde{E}_{\mathcal{N}}-\Delta E_{\mathcal{N}}>0$, which translates into $\left|r-r_{\text {crit }}\right|>\ln \left(2 \Delta x_{+} \Delta p_{+}\right) / 2=S_{r}$. Then, there is a phase where entanglement undergoes an infinite sequence of events of "sudden death" and "sudden revival" [26, 27]. This occurs if the initial state is such that $\left|\ln \left(1 / 2 m \Omega_{-} \Delta^{2} x_{+}\right) / 2\right| \leq r \leq \ln \left(2 \Delta^{2} p_{+} / m \Omega_{-}\right) / 2$. We denote this phase as "SDR" (for sudden death and revival). Finally, a third phase characterized by a final event of "sudden death" of entanglement may be realized if $r \leq-E_{c} \equiv-\ln \left(1 / 2 m \Omega_{-} \Delta^{2} x_{+}\right) / 2$. This phase is simply denoted as "SD" (for sudden death).

Depending on the properties of the environment (initial temperature, damping rate, etc) a given initial state (parameterized by the squeezing $r$ ) will belong to one of the three phases. For the ohmic environment we can use exact analytic expressions for the coefficients $D$ and $f$ [20] to obtain the phase diagram displayed in Fig. 1. In the diagram, the areas corresponding to each of the three phases are displayed. As a reference, we also include the curves that show the temperature dependence of $S_{r}$ and $r_{\text {crit }}$ (dashed and dotted lines respectively).

The phase diagram provides complete information about the asymptotic behavior of entanglement. It is worth mentioning some features of the diagram. The NSD phase present at low temperatures is purely nonMarkovian and non-perturbative. Its area shrinks as the damping rate decreases. This phase includes the coherent states, that can become entangled at very low temperature. The zero temperature line is also interesting: It contains states in the NSD phase for small and large
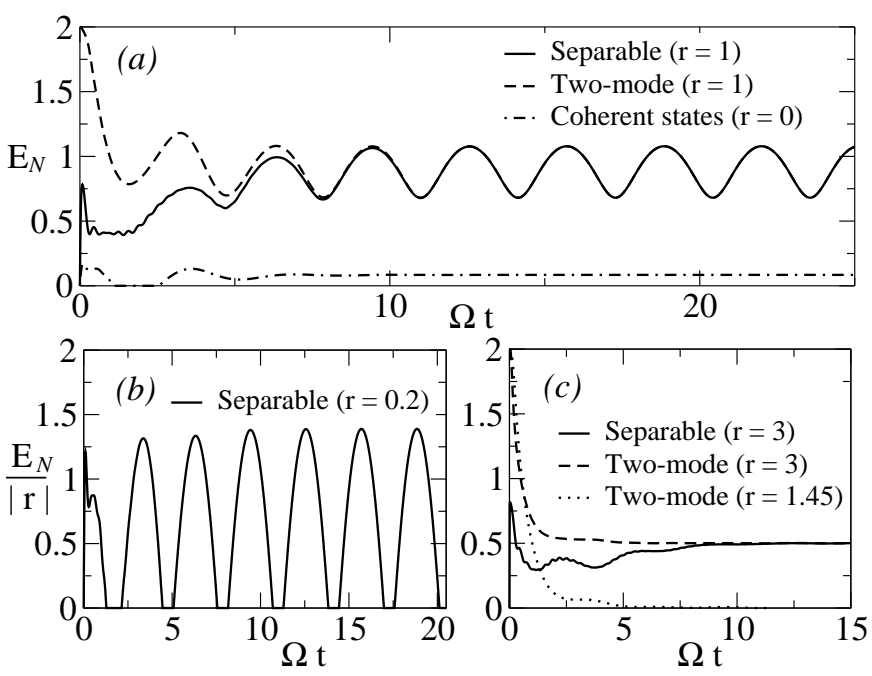

FIG. 2: Logarithmic negativity for resonant oscillators in the same environment. (a) For $T=0$ the NSD phase appears both for for large and small squeezing. Asymptotic behavior of initially entangled or separable states only depends on $r$. The amplitude of oscillations vanishes when $r \rightarrow 0$. (b) The SDR phase appears for intermediate values of squeezing at zero temperature. (c) $T / \Omega=10$, the SD phase appears for small $r$ and NSD phase for large squeezings, oscillations in the steady state are attenuated as the temperature increases.

squeezings. However for an intermediate range of squeezings centered about $r_{\text {crit }}$ states belong to the SDR phase.

To the contrary, the high temperature region of the diagram is quite different. Thus, for high temperatures we have $E_{c}<0$ (which implies that coherent states do not get entangled) and also $r_{\text {crit }} \rightarrow 0$ (which implies that the region covered by the SDR phase becomes narrower). Thus, for high temperatures initial states with large squeezing $\left(r>\ln \left(2 \Delta x_{+} \Delta p_{+}\right) / 2=S_{r}\right)$ retain some of their entanglement while those with squeezing factors smaller than the critical value $S_{r}$ suffer from sudden death. We remark that this is almost the same condition obtained previously for persistence of entanglement in the Markovian regime [9]. However, our analysis shows that the boundary between SD and NSD phases is rather subtle: for any finite temperature the two phases are separated by a very narrow portion of SDR phase (in this phase there are oscillations of the entanglement whose amplitude, $r_{c r i t}$ decreases as temperature grows). This is yet another interesting non-Markovian effect identified by our analysis. A final comment on the phase diagram: The NSD phase is characterized by a non-vanishing asymptotic entanglement that can be quantified in a straightforward way from the phase diagram itself. The average value of the logarithmic negativity is simply the distance to the dashed line (which signals the midpoint of the SDR phase) or just the distance between the dashed and dotted lines for $r \leq r_{\text {crit }}$.

The validity of the above rather simple but nontrivial 
analytical predictions can be verified by an exact numerical solution to the problem. We considered an ohmic environment with the following parameters: $\gamma_{0}=0.15$, $\Lambda=20, m=1, \Omega=1, C_{12}=0$ (extension to the case where the oscillators interact can be easily done). The results for the evolution of $E_{\mathcal{N}}$ are shown in Fig. 2. We clearly see that the final entanglement produced by different initial states only depends upon the squeezing factor $r$. The existence of events of sudden death (and revivals) can also be seen from the numerical solution, and is predicted from our analytic results characterizing the SDR phase, Fig. 2. It is also noticeable that the amplitude of the oscillations vanishes when $f / D \rightarrow 0$, which is a feature of the high temperature limit. The oscillating behavior of the final entanglement is a consequence of the non-zero asymptotic value of the anomalous diffusion coefficient $f(t)$, (see Fig. 2). Moreover, this same coefficient is also responsible for the generation of asymptotic entanglement in initial coherent states. But in this case the entanglement is constant $E_{\mathcal{N}}=E_{c}$ and increases with $\gamma_{0}$, as the final state of the oscillator $x_{+}$becomes more and more localized in position. This is another purely non-Markovian effect that is entirely lost in the RWA-Markovian approximation [9]. With the numerical solution, we verified that every possible temporal evolution that can be obtained by changing parameters fits either in the NSD, SDR or the SD phase. The boundaries between phases is also accurately described by the conditions mentioned above.

The above properties are valid under a single important assumption: the two oscillators are resonant. If this is not the case the analysis becomes more complicated: the master equation is no longer valid since there are terms coupling the $x_{-}$and $x_{+}$virtual oscillators. Due to this coupling the $x_{-}$oscillator is not protected from the environment and also reaches an equilibrium state. In Fig. 3 we show how things change when we move away from the resonance condition. It is clear that $E_{\mathcal{N}}$ decays much faster in this case. Also, the value of $E_{\mathcal{N}}$ was analyzed away from resonance for different times. The result is shown in Fig. 3 where it is clear that the resonance peak becomes sharper and sharper as time grows.

Our results provide a complete picture of the possible behavior of entanglement for resonant oscillators coupled to a common environment. The same analysis is valid for any environment such that an asymptotic value of the coefficients of the master equation is obtained. For example, for super-ohmic environments the damping coefficient approaches a small value while both $f(t)$ and $D(t)$ vanish asymptotically. Our analysis predicts that there will not be an equilibrium value for the dispersions of the $x_{+}$oscillator and that entanglement will oscillate approximately recovering its initial value after some time. This is indeed observed in our numerical simulations (contrary to what is indicated in [13], where the results obtained seem to be an artifact produced by an unreasonably low

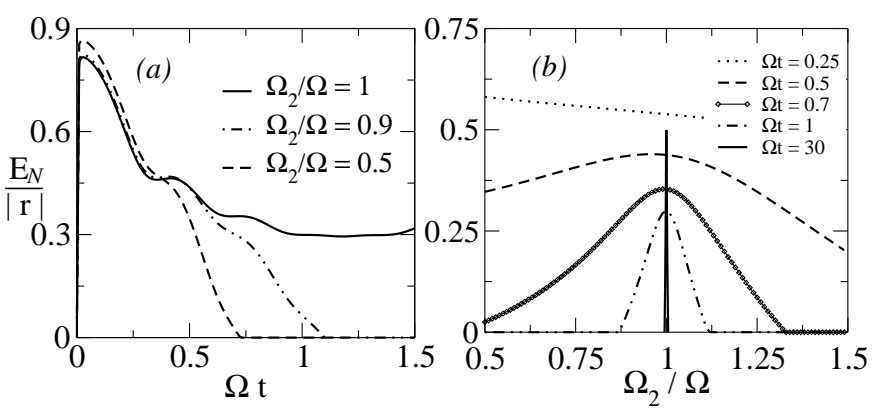

FIG. 3: Entanglement dynamics for non-resonant oscillators initially in a squeezed separable state $(r=3)$ and $T / \Omega=10$. (a) Entanglement is created between non-resonant oscillators but it banishes in finite time. (b) $E_{\mathcal{N}}$ for different times as a function of the frequency of the second oscillator, the resonant condition is essential for asymptotic entanglement.

cutoff). Finally, we remark once again that our results for the asymptotic entanglement are highly dependent on the validity of the resonance condition. For non-resonant oscillators the generic fate for long times is disentanglement due to the interaction with the environment. This issue will be analyzed in detail elsewhere. Authors acknowledge support from CONICET and Anpcyt (Argentina).

[1] D. Braun, Phys. Rev. Lett 89, 277901 (2002).

[2] F. Benatti, R. Floreanini, and M. Piani, Phys. Rev. Lett 91, 70402 (2003).

[3] S. Oh and J. Kim, Phys. Rev. A 73, 062306 (2006).

[4] C. Anastopoulos, S. Shresta, and B. L. Hu, arXiv:quant$\mathrm{ph} / 0610007$ (2006).

[5] T. Yu and J. H. Eberly, Phys. Rev. Lett 93, 140404 (2004)

[6] M. G. Paris, J. Opt. B 4, 442 (2002).

[7] A. Serafini, F. Illuminati, M. G. A. Paris, and S. De Siena, Phys. Rev. A 69, 022318 (2004).

[8] S. Maniscalco, S. Olivares, and M. G. A. Paris, Phys. Rev. A 75, 062119 (2007).

[9] J. S. Prauzner-Bechcicki, J. Phys. A: Math. Gen. 37, 173 (2004).

[10] P. J. Dodd and J. J. Halliwell, Phys. Rev. A 69, 052105 (2004).

[11] F. Benatti and R. Floreanini, J. Phys. A: Math. Gen. 39, 2689 (2006).

[12] K.-L. Liu and H.-S. Goan, Phys. Rev. A 76, 022312 (2007).

[13] J.-H. An and W.-M. Zhang, Phys. Rev. A 76, 042127 (2007).

[14] C. Hörhammer and H. Büttner, arXiv:0708.2670 (2007).

[15] B. L. Hu, J. P. Paz, and Y. Zhang, Phys. Rev. D 45, 2843 (1992).

[16] R. P. Feynman and F. L. Vernon, Ann. Phys. 24, 118 (1963).

[17] A. O. Caldeira and A. J. Leggett, Physica A 121, 587 (1983).

[18] C.-H. Chou, T. Yu, and B. L. Hu, arXiv:quant- 
ph/0703088 (2007).

[19] J. Eisert and M. B. Plenio, Phys. Rev. Lett. 89, 137902 (2002).

[20] C. H. Fleming, B. L. Hu, and A. Roura, arXiv:0705.2766 (2007).

[21] G. Vidal and R. F. Werner, Phys. Rev. A 65, 032314 (2002).

[22] J. Eisert, Ph.D. thesis, University of Postdam (2001).

[23] G. Adesso and F. Illuminati, Phys. Rev. A 70, 022318 (2004).
[24] A. Einstein, B. Podolsky, and N. Rosen, Phys. Rev. 47, 777 (1935).

[25] Here we consider the case where $\delta x_{-} \delta p_{-}=1 / 2$. In general, we can apply the same results replacing $\Delta x_{+} \Delta p_{+} \rightarrow$ $2 \Delta x_{+} \Delta p_{+} \delta x_{-} \delta p_{-}$.

[26] M. Yönac, T. Yu, and J. H. Eberly, J. Phys. B 39, S621 (2006),

[27] M. Yönac, T. Yu, and J. H. Eberly, J. Phys. B 40, S45 (2007). 